\title{
Solving Sudoku with Consistency: A Visual and Interactive Approach
}

\author{
Ian Howell, ${ }^{1}$ Robert Woodward, ${ }^{1,2}$ Berthe Y. Choueiry, ${ }^{1}$ Christian Bessiere ${ }^{2}$ \\ ${ }^{1}$ Constraint Systems Laboratory, University of Nebraska-Lincoln, USA \\ ${ }^{2}$ CNRS, University of Montpellier, France \\ \{ihowell|rwoodwar|choueiry\}@cse.unl.edu bessiere@lirmm.fr
}

\begin{abstract}
We describe an online, interactive system with a graphical interface to illustrate the power and operation of consistency algorithms in a friendly and popular context, namely, solving Sudoku puzzles. Our tool implements algorithms for enforcing five (domain-based) consistency properties on binary and non-binary constraint models. Our tool is useful for research, education, and outreach. From a scientific standpoint, we propose a new consistency property that can solve the hardest known $9 \times 9 \mathrm{Su}-$ doku instances without search, but leave open the question of the lowest level of consistency needed to solve every $9 \times 9$ Sudoku puzzle. We have used the current tool in the classroom to introduce students to modeling problems with constraints, explain consistency properties, and illustrate the operations of constraint propagation and lookahead. Finally, we have also used this tool during outreach activities to demystify AI to children and the general public and show them how computers 'think.'
\end{abstract}

\section{Introduction}

The popularity of the Sudoku puzzle stems perhaps from the simplicity of its rules and the wide range of difficulty levels in which it can found in magazines, books, and on the internet. This combinatorial decision problem is known to be NP-complete [Yato, 2003] and lends itself particularly well to introduce the general public and students of Computer Science to the area of Constraint Processing. The current state of the art in terms of the availability of online solvers (based on backtrack search or on convoluted patterns and rules defined by humans) has not changed much since 2007 when our lab introduced our first constraint-based Sudoku solver [Reeson et al., 2007]. Two references are worth mentioning: the first paper on using CP to solve Sudoku [Simonis, 2005] and a file with 375 instances of the hardest known Sudoku puzzles. ${ }^{1}$ Similarly to the approach of Simonis [2005] but using 'standard' consistency properties, we investigate the weakest level of consistency that is needed to solve a given Sudoku instance without search. In particular, we introduce a new consistency

\footnotetext{
${ }^{1}$ http://www.mediafire.com/?9ypndha1zadpwaw
}

property, and implement its algorithm, that can 'solve' the hardest known Sudoku instances without search (i.e., reduces to a singleton set the domains of all the variables of a $9 \times 9$ Sudoku instance that has exactly one solution).

Our tool is built with the web technologies of HTML, CSS, and JavaScript. ${ }^{2}$ It uses a database of Sudoku instances with meta data such as the number of clues and the weakest level of consistency needed to solve the instance without search. It implements algorithms for enforcing five consistency properties and visualization mechanisms to support explanation.

\section{Constraint Models and Consistency Properties}

Constraint Satisfaction Problems (CSPs) are defined as a tuple $(X, D, C)$, where $X$ is a set of variables, $D$ is the set of domains of the variables with $D_{i}$ representing the domain of $X_{i}$, and $C$ is a set of constraints, where a constraint $C_{i}$ has scope $\left\{x_{i_{1}}, \ldots, x_{i_{k}}\right\}$ and a set of acceptable assignments $\left(v_{i_{1}}, \ldots, v_{i_{k}}\right) \in D_{i_{1}} \times \ldots \times D_{i_{k}}$ resticting the values assignable to the variables in $C_{i}$ 's scope. In a binary CSP, a constraint has two variables in its scope. In non-binary CSPs, it has more. Solving a CSP requires assigning a value to each variable such that all constraints are satisfied.

The constraint model of the $9 \times 9$ Sudoku puzzle has 81 variables representing the cells of the $9 \times 9$ board. The domain of a variable is the set $\{1,2, \ldots, 9\}$. The binary model has 810 binary DIFF constraints defined over every two variables in the same row, column, or block. The non-binary model has 27 non-binary ALLDIFFERENT constraints defined over the 9 variables appearing in a row, column, or block [Régin, 1994].

Consistency algorithms enforce a given (domain-based) consistency property by removing, from the domains, values that cannot appear in a solution to the CSP. Such algorithms are typically used before search and/or as lookahead during search to reduce the size of the search space. Below, we distinguish between the consistency properties of the binary model and those of the non-binary model by including the letter $\mathrm{G}$ for the latter (e.g., AC and GAC) and state the definitions in a way that is applicable to both models.

Definition 1 Arc Consistency (AC and GAC) [Waltz, 1975] [Mackworth, 1977]: A constraint network $P=(X, D, C)$ is

\footnotetext{
${ }^{2}$ http://sudoku.unl.edu/
} 
$A C$ iff, for every constraint $C_{i} \in C$, and $\forall x_{j} \in \operatorname{scope}\left(C_{i}\right)$, every value $v \in D\left(x_{j}\right)$ is consistent with $C_{i}$ (i.e., appears in some support of $C_{i}$ ).

Definition 2 Singleton Arc Consistency (SAC and SGAC) [Debruyne and Bessiere, 1997]: A constraint network $P=$ $(X, D, C)$ is singleton arc consistent iff $\forall x_{i} \in X, \forall a \in$ $D\left(x_{i}\right)$, the network $\left.P\right|_{x_{i}=a}=\left(X,\left.D\right|_{x_{i}=a}, C\right)$ obtained by re-placing $D\left(x_{i}\right)$ by the singleton $\{a\}$ is not arc inconsistent. If $\left.P\right|_{x_{i}=a}$ is arc inconsistent, we say that $\left(x_{i}, a\right)$ is SAC inconsistent.

Definition 3 Partition-One Arc Consistency (POAC and POGAC) [Bennaceur and Affane, 2001]: Given a constraint network $P=(X, D, C)$, a variable $x_{i}$ is partition-one-AC iff $D\left(x_{i}\right) \neq \emptyset$, all values in $D\left(x_{i}\right)$ are $S A C$, and $\forall j \in$ $\{1 \ldots n\}, j \neq i, \forall v_{j} \in D\left(x_{j}\right), \exists v_{i} \in D\left(x_{i}\right)$ such that $v_{j} \in A C\left(\left.P\right|_{s_{i}=v_{i}}\right)$. The constraint network $P$ is POAC iff all its variables are POAC.

Definition 4 Bidirectional Singleton Arc Consistency (BiSAC and BiSGAC) [Bessiere and Debruyne, 2008]: $A$ constraint network $P=(X, D, C)$ is bidirectional singleton arc consistent iff $\forall x_{i} \in X, \forall a \in D_{i}, A C\left(T_{i a}\right) \neq \emptyset$, where $T_{i a}=\left(X, D_{i a}^{T}, C\right)$, with $D_{i a}^{T}\left(x_{j}\right)=\left\{b \in D\left(x_{j}\right) \mid\left(x_{i}, a\right) \in\right.$ $\left.A C\left(\left.P\right|_{x_{j}=b}\right)\right\}$.

Definition 5 Double Singleton Arc Consistency (SSAC and SSGAC): A constraint network $P=(X, D, C)$ is double singleton arc consistent iff $\forall x_{i} \in X, \forall a \in D\left(x_{i}\right)$ the network $\left.P\right|_{x_{i}=a}=\left(X,\left.D\right|_{x_{i}=a}, C\right)$ obtained by replacing $D\left(x_{i}\right)$ by the singleton $\{a\}$ is not singleton arc inconsistent.

Of the above properties, AC is the weakest and SSGAC is the strongest. To enforce GAC, SGAC, POGAC, BiSGAC and SSGAC, we use the specialized algorithm for the ALLDIFFERENT global constraint [Régin, 1994]. We also implement a backtrack search for finding all solutions, in the case the puzzle entered has more than one solution (i.e., ill formed).

A $9 \times 9$ well-formed Sudoku puzzle has a fixed size and, by definition, a single solution. Thus, the question of the weakest consistency to solve every instance is legitimate but remains open. Interestingly, SSGAC solves all 375 hardest known Sudoku instances. In fact, 276 instances require at least SSGAC, 25 BiSGAC+SSAC, 7 BiSGAC, and 67 SSAC.

\section{User Interface}

The user interface has two halves: the control panel to the right (Figure 1) and the Sudoku board to the left (Figure 2).

The control panel has five tabs to access various functionalities: LOAD (an instance from the database), UPLOAD (a picture of an instance using OCR methods from Aruco and CV Javascript libraries), SOLVE (the current instance on the board), and SUBMIT (a new instance to the database).

The board shows a standard $9 \times 9$ Sudoku layout. It is surrounded on the top and left sides by characters designating columns and rows and on the bottom and right sides by buttons to enforces AC (single line) and GAC (multiple lines) on the variables of the corresponding row or column.

The board reflects the current state of the constraint model. Clues are displayed as blue characters in bold and are immutable but can be converted to standard assigned values.

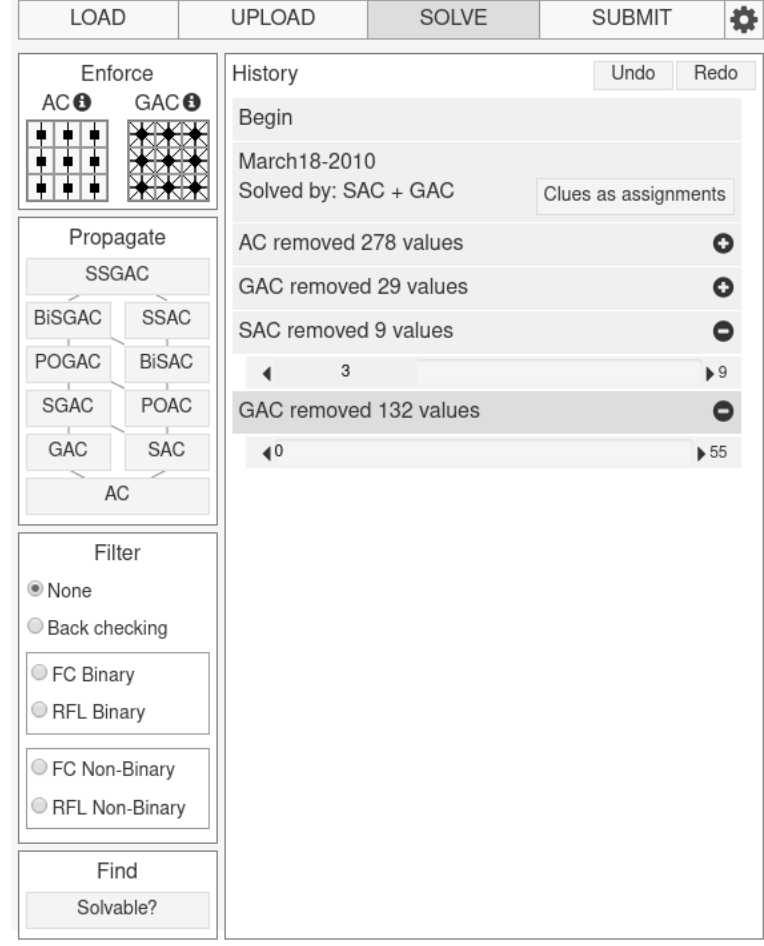

Figure 1: Enforcing the sequence AC, GAC, SAC, then GAC in the SOLVE tab of the control panel

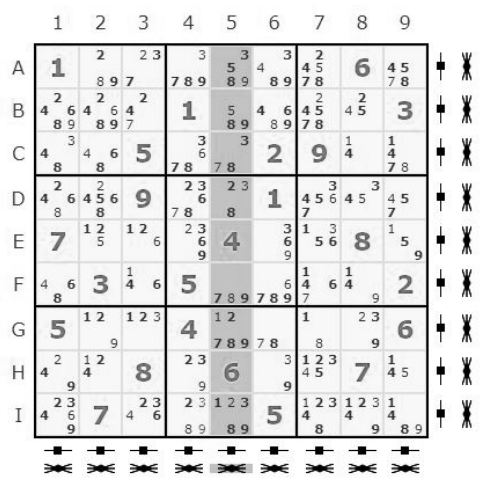

Figure 2: The Sudoku board

From the SOLVE tab, the user can apply AC or GAC on a block (ENFORCE), enforce one of the 10 consistencies shown in a Hasse diagram of their relative strength (PROPAGATE), maintain partial or realfull lookahead while interactively instantiating variables (FILTER), run search to find all the solutions of the configuration on the board (FIND). As the user enforces a consistency property (PROPAGATE), the board is dynamically updated to reflect the effects of this action. The user can step through the iterations of a given consistency algorithm. The board is animated by highlighting in grey the constraint under consideration, in red values removed at the current iteration, and bolded in black the values to be removed in future iterations of the same propagation.

\section{Acknowledgments}

This research is supported by an NSF Grant No. RI-1619344 and a UNL UCARE grant. 


\section{References}

[Bennaceur and Affane, 2001] Hachemi Bennaceur and Mohamed-Salah Affane. Partition-k-AC: An Efficient Filtering Technique Combining Domain Partition and Arc Consistency. In Principles and Practice of Constraint Programming (CP 01), volume 2239 of LNCS, pages 560-564. Springer, 2001.

[Bessiere and Debruyne, 2008] Christian Bessiere and Romuald Debruyne. Theoretical analysis of singleton arc consistency and its extensions. Artificial Intelligence, 172(1):29-41, 2008.

[Debruyne and Bessiere, 1997] Romuald Debruyne and Christian Bessiere. Some practicable filtering techniques for the constraint satisfaction problem. In In Proceedings of IJCAI'97, pages 412-417, 1997.

[Mackworth, 1977] Alan K. Mackworth. Consistency in Networks of Relations. Artificial Intelligence, 8:99-118, 1977.

[Reeson et al., 2007] Christopher G. Reeson, Kai-Chen Huang, Kenneth M. Bayer, and Berthe Y. Choueiry. An Interactive Constraint-Based Approach to Sudoku. In Proceedings of AAAI-2007, pages 1976-1977, 2007.

[Régin, 1994] Jean-Charles Régin. A filtering algorithm for constraints of difference in constraint satisfaction problems. In Proceedings of AAAI-94, pages 362-437, Seattle, WA, 1994.

[Simonis, 2005] Helmut Simonis. Sudoku as a constraint problem. In Proceedings of the Fifth International Workshop on Modeling and Reformulating Constraint Satisfaction Problems, pages 13-27, 2005.

[Waltz, 1975] David Waltz. Understanding Line Drawings of Scenes with Shadows. In P.H. Winston, editor, The Psychology of Computer Vision, pages 19-91. McGraw-Hill, Inc., 1975.

[Yato, 2003] Takayuki Yato. Complexity and Completeness of Finding Another Solution and its Application to Puzzles. Master's thesis, University of Tokyo, 2003. 